Ultrasound in Med. \& Biol., Vol. 00, No. 00, p. 1, 2020 Copyright $\odot 2020$ World Federation for Ultrasound in Medicine \& Biology. All rights reserved. Printed in the USA. All rights reserved. $0301-5629 / \$$ - see front matter

\title{
- Letter to the Editor
}

\section{HUNTING THE VULNERABLE CAROTID PLAQUE: IN SEARCH OF A GOLD STANDARD}

\author{
(Received 15 July 2020; In final from 15 July 2020)
}

To the Editor:- Vrachatis et al. demonstrated particular interest in our recent review of the literature on the strengths and limitations of contrast-enhanced ultrasound (CEUS) for the detection of carotid intraplaque neovascularization (Schinkel et al. 2020). I am pleased to have the opportunity to concisely address the two themes they highlighted: the standardization of methods to detect and quantify intraplaque neovascularization, and the correlation of CEUS findings with histopathological results.

First, an overview of the quantification methods that were used in the 52 available studies including a total of 4660 patients is provided in Table 1 of the review. Several software packages, which were described in more detail in the review, were developed for quantification of carotid intraplaque neovascularization. To provide more homogeneous data, future studies should preferably use a standardized protocol for reporting methodology and results; this would facilitate comparison of the individual studies.

Second, an overview of 17 studies including a total of 576 patients on the validation of intraplaque neovascularization on CEUS with histopathological results was provided in Table 2. There are several causes why CEUS findings do not perfectly correlate with histopathological results. Carotid CEUS consists of a dynamic examination evaluating plaque perfusion, whereas histopathological analysis merely assesses the presence of microvessels, providing no information on perfusion status. Next, the available studies have included patients with advanced carotid disease with an indication for carotid surgery, causing a selection bias. The vast majority of patients who undergo carotid CEUS do not have an indication for carotid surgery, and thus, histology cannot be obtained. During carotid surgery, the plaque is resected en bloc if possible; however, peri-operative conditions may limit the vascular surgeon, and the plaque is removed in fragments, making comparison with imaging findings challenging. Finally, systematic sampling errors occur: CEUS clips are acquired in a longitudinal direction of the carotid artery, whereas histopathological analysis is performed on cross-sectional samples, hindering a correlation analysis. Because of these limitations, histology is, on closer study, not the ideal reference technique or so-called gold standard that it seems to be.

Conflict of interest disclosure: None

AREND F.L. SCHINKEL Department of Cardiology, Erasmus Medical Center, Rotterdam, The Netherlands

Address correspondence to Arend F.L. Schinkel, Department of Cardiology, Erasmus Medical Center, 's-Gravendijkwal 230, Rotterdam, Zuid-Holland 3015CE, The Netherlands.

\section{REFERENCE}

Schinkel AFL, Bosch JG, Staub D, Adam D, Feinstein SB. Contrastenhanced ultrasound to assess carotid intraplaque neovascularization. Ultrasound Med Biol 2020;46:466-478. 